\title{
The effect of postoperative infection on survival in patients with glioblastoma
}

\author{
Yi-Ren Chen, MD, MPH, ${ }^{1}$ Beatrice Ugiliweneza, PhD, MS, ${ }^{2}$ Eric Burton, MD, ${ }^{3}$ Shiao Y. Woo, MD, ${ }^{4}$ \\ Maxwell Boakye, MD, MPH, ${ }^{2}$ and Stephen Skirboll, MD ${ }^{1,5}$

\begin{abstract}
${ }^{1}$ Department of Neurosurgery, Stanford University, Palo Alto, California; Departments of ${ }^{2}$ Neurosurgery, ${ }^{3}$ Neurology, and
\end{abstract} \\ ${ }^{4}$ Radiation Oncology, University of Louisville, Kentucky; and ${ }^{5}$ Section of Neurosurgery, VA Palo Alto Health Care System, \\ Palo Alto, California
}

\begin{abstract}
OBJECTIVE Glioblastoma is a primary glial neoplasm with a median survival of approximately 1 year. There are anecdotal reports that postoperative infection may confer a survival advantage in patients with glioblastoma. However, only a few case reports in the literature, along with 2 retrospective cohort studies, show some potential link between infection and prolonged survival in patients with glioblastoma. The objective of this study was to evaluate the effect of postoperative infection in patients with glioblastoma using a large national database.
\end{abstract}

METHODS The linked Surveillance, Epidemiology, and End Results (SEER)-Medicare database was searched to identify patients 66 years of age and older with glioblastoma, with and without infection, from 1997 to 2010 . The primary outcome was survival after diagnosis. The statistical analysis was performed with a graphical representation using KaplanMeier curves, univariate analysis with the log-rank test, and multivariate analysis with proportional hazards modeling.

RESULTS A total of 3784 patients with glioblastoma were identified from the database, and from these, 369 (9.8\%) had postoperative infection within 1 month of surgery. In patients with glioblastoma who had an infection within 1 month of surgery, there was no significant difference in survival (median 5 months) compared with patients with no infection (median 6 months; $p=0.17$ ). The study also showed that older age, increased Gagne comorbidity score, and having diabetes may be negatively associated with survival.

CONCLUSIONS Infection after craniotomy within 1 month was not associated with a survival benefit in patients with glioblastoma.

https://thejns.org/doi/abs/10.3171/2016.8.JNS16836

KEY WORDS glioblastoma; postoperative infection; outcome; SEER Medicare database; oncology

$\mathrm{G}$ LIOBLASTOMA has one of the worst prognoses of any neoplasm, with a median overall survival of 6-14 months and a 2-year survival rate of $15 \%-26 \% .{ }^{16,17}$ Factors that are correlated with survival include tumor size and location (supratentorial and cerebellar), age at presentation, Karnofsky Performance Scale (KPS) score at presentation, treatment modality, histological findings, and $\mathrm{O}^{6}$-methylguanine-DNA methyltransferase (MGMT) methylation status. ${ }^{18,20}$ Over the years, there have been several anecdotal reports of postoperative infection conferring improved survival in patients with glioblastoma, but there have been few studies providing data to substantiate this claim. There have been 2 retrospective studies investigating the effect of postoperative infections on survival in patients with glioblastoma. Bohman et al. ${ }^{1}$ conducted a single-center study over 10 years examining 18 patients with bacterial infections. After matching these patients with age-matched cohorts, they showed no survival advantage in patients with postoperative infections. Another study by De Bonis et al. ${ }^{3}$ investigated 10 patients with postoperative bacterial infections. Multivariate analysis showed that the infection group had a median survival of 30 months, compared with 15 months for patients without infection.

Given the lack of published research, as well as the

ABBREVIATIONS $\mathrm{Cl}=$ confidence interval; $\mathrm{HCMV}=$ human cytomegalovirus; $\mathrm{HR}=$ hazard ratio; $I \mathrm{QR}$ = interquartile range; $\mathrm{KPS}=$ Karnofsky Performance Scale; $\mathrm{MGMT}=$ O6-methylguanine-DNA methyltransferase; SEER = Surveillance, Epidemiology, and End Results.

SUBMITTED April 3, 2016. ACCEPTED August 8, 2016.

INCLUDE WHEN CITING Published online December 9, 2016; DOI: 10.3171/2016.8.JNS16836. 
small sample sizes available in the current retrospective studies, the aim of our study was to provide a more comprehensive investigation of the subject using the Surveillance, Epidemiology, and End Results (SEER)-Medicare database, which provides more nationally representative data and a larger sample size. Our intent was to perform a retrospective comparison of patients with glioblastoma with or without postoperative infections and assess its impact on survival.

\section{Methods \\ Data Source}

We used the linked database SEER-Medicare. SEER is a national database that includes cancer cases with cancer details (such as tumor details, first course of treatment, number of cancers, survival, mortality and cause, etc.) along with patient characteristics and demographics. The Medicare part contains different claims of different health services used with associated payments. Thus, the SEERMedicare database is restricted to data of the elderly (age 65 or older) with cancer. Each patient in the database has a unique identifier that is used to link to different data files. SEER-Medicare is available, for a fee, to researchers on a project-to-project basis.

Our SEER-Medicare is a tailored database, containing patients with primary brain tumors from 1997 to 2010 with their Medicare claims. Our Medicare part is composed of the Medicare Provider Analysis and Review file, the outpatient claims file, the Physician/Supplier Part B file, the Durable Medical Equipment file, and the Hospice file.

\section{Inclusion and Exclusion Criteria and Patient Selection}

The inclusion criteria of the study were as follows: 1 ) histology codes $94403,9441 / 3$, or $9442 / 3$, with concurrent topology codes $\mathrm{C} 71.0-\mathrm{C} 71.4 ; 2$ ) age 66 years or older at diagnosis to allow for 1 full year retrospective review; 3 ) microscopically confirmed resection-craniotomy (ICD-9 codes 01.24, 01.25, 01.31, 01.32, 01.39, 01.51, 01.53, 01.59; CPT code 61304) or biopsy (ICD-9 codes 01.11-01.14, 01.18; CPT4 code 61140); 4) having both part A and part B Medicare during the period from 1 month before diagnosis until death or until December 31, 2010; and 5) having age as the only reason for Medicare entitlement.

The exclusion criteria were: 1) a diagnosis of other primary tumors within 1 year of diagnosis; 2) not having surgery; or 3) unknown or missing surgery status according to SEER. Also, patients for whom the surgery or biopsy was not found in the Medicare part in the period from 15 days before the diagnosis date to 2 months after were excluded. Enrollment in a health maintenance organization (HMO) at any time from 1 month before diagnosis until death or December 31, 2010, was also cause for exclusion.

The time of diagnosis was obtained from the SEER database. The SEER database dates only contain the month and the year. Therefore, we assumed the day of diagnosis as the 15th day of the month. Then, we adjusted all the analyses to take into account this 15-day error.

Patient characteristics were mainly obtained from the SEER database. Characteristics considered were the age at diagnosis, the race and sex of the patient, and the extent of surgery. Some characteristics were extracted from the Medicare part. Characteristics included were comorbidities such as the Gagne score, having diabetes, smoking status, having prior craniotomy, and whether a patient underwent a reoperation within 30 days or had complications within 30 days of diagnosis. The Gagne score is a comorbidity score that was developed to include both the Charlson score and the Elixhauser score. It was found to outperform each of the other scores individually. ${ }^{4}$ Smoking status was recognized by the presence of ICD-9 code 305.1 and diabetes by ICD- 9 code 250 .

\section{Explanatory Variables and Outcome Variable}

The testing variable was whether the patient developed infection or not. We considered bacterial meningitis (ICD-9 codes 320-320.9), nonbacterial meningitis (ICD-9 codes 321-321.8, 322-322.9), intracranial abscess (ICD9 code 324.0), and postoperative infection (ICD-9 codes 998.5, 998.59, and 998.51) as positive for infection. Other explanatory variables were patient characteristics and demographics described above. The outcome variable was survival. Survival was counted in months from 15 days from diagnosis until death or until censoring on December $31,2010$.

\section{Statistical Analysis}

Data were summarized using means and standard deviations as well as medians and interquartile ranges (IQRs) for continuous variables, and number and percentage for categorical variables. Patient characteristics and demographics were compared with the Wilcoxon rank-sum test for continuous variables and the chi-square test for categorical variables.

The impact of infection on survival was first assessed using univariate analysis. Kaplan-Meier curves were used to display survival, then the survival times were compared using the log-rank test. A multivariate analysis was also performed using the proportional hazards method, with infection status as the testing variable and controlling for age, race, sex, extent of initial surgery, Gagne score, diabetes, smoking status, and whether the patient had previous craniotomies. All tests were 2 -sided and a p value $<0.05$ was considered significant. All data preprocessing and analysis was performed in SAS 9.4 (SAS Institute).

\section{Results}

Using the SEER-Medicare database, a total of 3784 patients with glioblastoma from 1997 to 2010 were identified. Of these patients, 3670 had at least 1 month of survival. One hundred fifty-four patients $(4.2 \%)$ had infection (defined as bacterial or nonbacterial meningitis, intracranial abscess, or postoperative infection by ICD-9 coding) within the 1st month of surgery. Postoperatively, 3516 (96\%) had no evidence of any infection. When compared with patients with no infections, patients who had infections had no significant difference in age, Gagne comorbidity score, race, extent of surgery (defined by local, partial, or gross-total resection), diabetic status, and smoking status (Table 1). There was a lower percentage of patients in the 
TABLE 1. Effect of infection within 1 month after glioblastoma surgery on survival

\begin{tabular}{|c|c|c|c|}
\hline \multirow[b]{2}{*}{ Variable } & \multicolumn{3}{|c|}{ Infections w/in 1 Mo of Surgery } \\
\hline & No & Yes & $p$ Value \\
\hline No. of patients & 3516 & 154 & \\
\hline \multicolumn{4}{|l|}{ Age in yrs } \\
\hline Mean (SD) & $74.63(5.77)$ & $74.62(5.42)$ & \\
\hline Median (IQR) & $74(70-78)$ & $74(70-78)$ & 0.8287 \\
\hline Age categories in yrs (\%) & & & 0.8605 \\
\hline $66-69$ & $779(22.16)$ & $36(23.38)$ & \\
\hline $70-74$ & $1065(30.29)$ & $42(27.27)$ & \\
\hline $75-79$ & $962(27.36)$ & $45(29.22)$ & \\
\hline $80-84$ & $505(14.36)$ & $24(15.58)$ & \\
\hline$\geq 85$ & $205(5.83)$ & $7(4.55)$ & \\
\hline \multicolumn{4}{|l|}{ Gagne comorbidity score } \\
\hline Mean (SD) & $1.26(1.14)$ & $1.51(1.36)$ & \\
\hline Median (IQR) & $1.00(0.50-1.75)$ & $1.11(0.60-1.91)$ & 0.0640 \\
\hline Females (\%) & $1662(47.27)$ & $57(37.01)$ & 0.0125 \\
\hline Race (\%) & & & 0.1815 \\
\hline White & $3278(93.23)$ & $140(90.91)$ & \\
\hline Black & $110(3.13)$ & $4(2.60)$ & \\
\hline Other & $128(3.64)$ & $10(6.49)$ & \\
\hline Extent of surgery (\%) & & & 0.2616 \\
\hline Local excision & $911(25.91)$ & $39(25.32)$ & \\
\hline Partial resection & $1204(34.24)$ & $50(32.47)$ & \\
\hline Gross-total resection & $1326(37.71)$ & $58(37.66)$ & \\
\hline All other extent & $75(2.13)$ & $7(4.55)$ & \\
\hline Had diabetes (\%) & $574(16.33)$ & $25(16.23)$ & 0.9736 \\
\hline Smoked (\%) & $148(4.21)$ & $2(1.30)$ & 0.0740 \\
\hline
\end{tabular}

infection group that was female at $37.0 \%$, compared with $47.3 \%$ in the no-infection group $(\mathrm{p}=0.013)$.

Having an infection did not appear to affect survival in patients who experienced an infection within 1 month of glioblastoma resection, with a median survival of 5 months in the infection group compared with 6 months in the no-infection group, resulting in a hazard ratio (HR) of 1.114 (95\% confidence interval [CI] 0.945-1.313), and a $\mathrm{p}$ value of 0.17 (Table 2). Age (HR 1.050), average Gagne comorbidity score (HR 1.063), and having diabetes (HR 1.175) did appear to correlate with worse survival, whereas extent of surgery, race, smoking status, and whether the patient had previously undergone a craniotomy did not affect survival (Table 2). The Kaplan-Meier survival curve is shown in Fig. 1.

\section{Discussion}

Although postoperative infections have been considered historically to have a negative impact on both morbidity and mortality in surgical patients, anecdotal evidence, as well as recent case reports and retrospective studies, have indicated the possibility that postoperative infection in patients with glioblastoma may have a protective effect on long-term survival. A literature search found 9 patients in case reports or case series with intracranial tumors that had prolonged survival associated with postoperative infections..$^{2,8,11,12,19}$ Of these case reports, only 2 patients had glioblastoma, with others having high-grade (III or above) astrocytomas.

Prior to our study, there have only been 3 retrospective cohort studies investigating the impact of postoperative infection on survival in patients with glioblastoma. In 2009, Bohman et al. conducted the first single-center review of 382 patients with glioblastoma and assessed the impact of postoperative infection on survival. ${ }^{1}$ Eighteen patients had bacterial infections, and of these, 17 had gram-positive infections. These cases were then compared with age-matched cohorts. Overall, a statistically insignificant survival advantage was observed in the infection group (Kaplan-Meier, $\mathrm{p}=0.27$ ). Interestingly, when patients whose infections occurred late in their courses were removed from the analysis, the advantage in mean survival disappeared. Early infection was defined in the paper as infections in the first half of the postoperative survival period compared with total survival time.

In 2011, De Bonis et al. studied 197 patients with glioblastoma and identified $10(5.08 \%)$ with postoperative bacterial infection. ${ }^{3}$ They showed that the infection group had a median survival of 30 months, compared with 15 months for patients without infection. They also showed 
TABLE 2. Effect on survival of infection within 1 month after glioblastoma surgery

\begin{tabular}{lc}
\hline \multicolumn{1}{c}{ Variable } & $\mathrm{HR}(95 \% \mathrm{Cl})$ \\
\hline Had infections w/in 1 mo & $1.114(0.945-1.313)$ \\
\hline Extent of initial surgery & Ref \\
\hline Local excision & $1.051(0.965-1.144)$ \\
\hline Partial resection & $0.779(0.716-0.847)$ \\
\hline Gross-total resection & $1.091(0.868-1.372)$ \\
\hline All other extent & Ref \\
\hline Race & $1.192(0.988-1.439)$ \\
\hline White & $0.889(0.749-1.055)$ \\
\hline Black & $1.050(1.044-1.057)$ \\
\hline Other & $1.063(1.035-1.092)$ \\
\hline Age (1-yr increase) & $1.175(1.075-1.284)$ \\
\hline Average Gagne comorbidity score for 6 & $1.168(0.989-1.379)$ \\
\hline mos prior to Dx (1-unit increase) & $1.018(0.587-1.765)$ \\
\hline Had diabetes
\end{tabular}

Dx $=$ diagnosis; Ref $=$ reference.

Boldface type indicates statistical significance.

that patients who underwent subtotal resection and had a KPS score of 70 or lower was negatively associated with survival.

In addition to the 2 retrospective studies on the effect of bacterial infection on survival in patients with glioblastoma, there was a study by Rahbar et al. in 2012 that examined the effect of low-grade human cytomegalovirus (HCMV) infection on survival. ${ }^{14}$ Brain tumor tissue sections from consecutive patients with glioblastoma who survived more than 18 months $(n=40)$ were examined. Sixteen $(40 \%)$ of the 40 patients with glioblastoma who lived more than 18 months had a low-grade HCMV infection, whereas only $3(8 \%)$ of 40 patients who lived less than 18 months did $(p=0.0006)$. The authors concluded that low-grade HCMV infection was strongly associated with long-term survival in patients with glioblastoma.

Our study is the first large-scale database study ( $\mathrm{n}=$ 3784) to examine the effect of postoperative infection on survival in patients with glioblastoma. In patients with glioblastoma who had infections within 1 month of surgery, there was no difference in survival between the infection (median survival 5 months) and no-infection (median 6 months) groups $(\mathrm{p}=0.17)$. We also showed that older age, increased Gagne comorbidity score, and having diabetes may be negatively associated with survival. Our results are consistent with those of the Bohman et al. study, which ultimately showed that postoperative infection did not confer any survival advantage in patients with glioblastoma. ${ }^{1}$

Although several studies have now shown that postoperative infection does not confer a survival benefit in patients with glioblastoma, there continues to be speculation in the neurosurgical community to the contrary. Several theories have been proposed in the past to explain why postoperative infection may lead to improved survival.

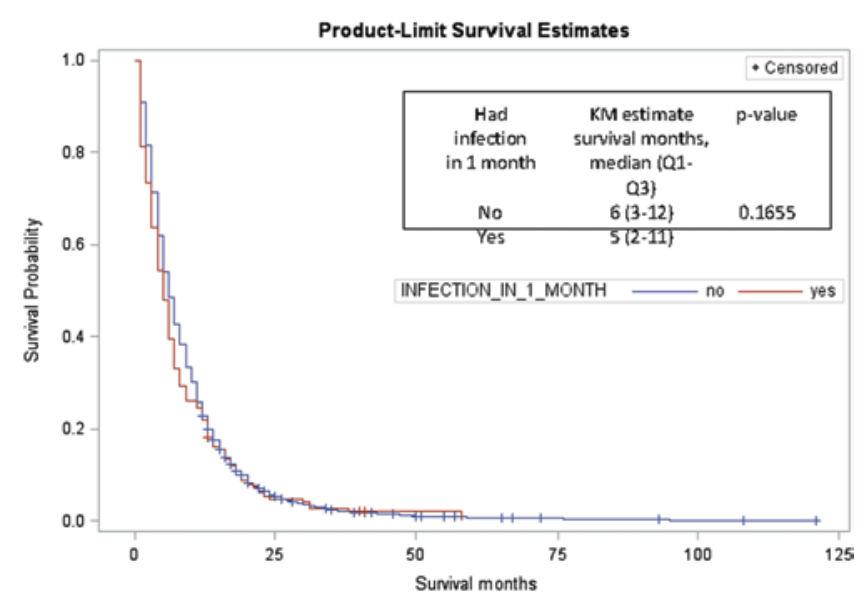

FIG. 1. Kaplan-Meier (KM) survival curve showing the effect of infection within 1 month of surgery. IQR =Q1-Q3. Figure is available in color online only.

De Bonis et al. ${ }^{3}$ theorized that it may be due to antagonistic proliferation of the bacteria, as in local competition for resources between the tumor cells and the replicating microorganisms. Another theory proposed by Bohman et al. ${ }^{1}$ and other authors is that infection in the tumor bed may stimulate the patient's immune response, thereby serving as a form of endogenous immunotherapy against glioblastoma cells. Additionally, Burt Vogelstein's laboratory reported an interesting study where they first injected CT26 (colon) and RENCA (renal carcinoma) into 47 mice, and then introduced the anaerobic bacterium Clostridium novyi-NT into the animals and found that $30 \%$ of mice treated with such spores were cured of their cancers. The authors also concluded that the effect was immune mediated, because cured animals rejected a subsequent challenge of the same tumor. ${ }^{16}$ In particular, other authors have postulated that tumor necrosis factor- $\alpha$, a factor secreted in response to infection, may be involved in cytotoxic cellmediated tumor suppression. $5,7,13$

Interestingly, there are also some other studies outside the neurosurgical literature pointing to a possible link between infection and survival in malignancies. Ruckdeschel et al. retrospectively reviewed 18 patients with lung carcinoma complicated by postoperative empyema. ${ }^{15}$ The 5 -year survival rate for the empyema group was $50 \%$, compared with $18 \%$ for the control group of 34 patients. Another study by Jeys et al. found that patients with osteosarcomas who had postoperative infection after endoprosthetic surgery had a survival rate of $84.5 \%$ at 10 years $(\mathrm{n}=41)$, compared with $62.3 \%$ in the noninfected group $(\mathrm{n}=371 ; \mathrm{p}=0.017)^{7}$. They showed that infection was an independent prognostic factor on Cox regression analysis. Finally, in the veterinary literature Lascelles et al. examined 47 dogs with osteosarcoma of the distal radius ${ }^{9}$ and showed that the dogs with an infection were less likely to die, with an HR of 0.446 .

There are several limitations of this study. The sensitivity and specificity of the patients identified in our study depend on the accuracy of input in the SEER-Medicare database; coding practices likely vary by hospital and various factors may influence coding. ${ }^{6,10}$ Additionally, the 
SEER-Medicare database did not have the granularity to allow us to break down infection status by deep versus superficial wound status, clinical biomarkers (MGMT promoter methylation status, $1 \mathrm{p} / 19 \mathrm{q}$ deletion status), or treatment with radiation therapy and/or chemotherapy, factors that may influence survival in patients with glioblastoma. ${ }^{1}$ The lack of granularity also makes it difficult to determine the specific organism causing the infection, or the specific type of postoperative infection, as we were only able to break down the infections into bacterial, nonbacterial, intracranial abscess, or postoperative infection by ICD-9 coding.

\section{Conclusions}

Despite its limitations, this study used the large number of records available in the SEER-Medicare database to provide a national view of the effect of postoperative infection on survival in patients with glioblastoma. Overall, there was no difference in survival for patients with glioblastoma who had infections within 1 month postoperatively. However, given the persistent interest in the possibility of postoperative infections conferring survival in patients with glioblastoma, as well as studies outside the neurosurgical literature showing potential survival benefit, further retrospective and even prospective large-scale studies are needed that allow for greater granularity, to further investigate this important question.

\section{References}

1. Bohman LE, Gallardo J, Hankinson TC, Waziri AE, Mandigo CE, McKhann GM II, et al: The survival impact of postoperative infection in patients with glioblastoma multiforme. Neurosurgery 64:828-835, 2009

2. Bowles AP Jr, Perkins E: Long-term remission of malignant brain tumors after intracranial infection: a report of four cases. Neurosurgery 44:636-643, 1999

3. De Bonis P, Albanese A, Lofrese G, de Waure C, Mangiola A, Pettorini BL, et al: Postoperative infection may influence survival in patients with glioblastoma: simply a myth? Neurosurgery 69:864-869, 2011

4. Gagne JJ, Glynn RJ, Avorn J, Levin R, Schneeweiss S: A combined comorbidity score predicted mortality in elderly patients better than existing scores. J Clin Epidemiol 64:749-759, 2011

5. Hibbs JB Jr, Lambert LH Jr, Remington JS: Resistance to murine tumors conferred by chronic infection with intracellular protozoa, Toxoplasma gondii and Besnoitia jellisoni. J Infect Dis 124:587-592, 1971

6. Hsia DC, Krushat WM, Fagan AB, Tebbutt JA, Kusserow RP: Accuracy of diagnostic coding for Medicare patients under the prospective-payment system. N Engl J Med 318:352-355, 1988

7. Jeys LM, Grimer RJ, Carter SR, Tillman RM, Abudu A: Post operative infection and increased survival in osteosarcoma patients: are they associated? Ann Surg Oncol 14:28872895, 2007

8. Kapp JP: Microorganisms as antineoplastic agents in CNS tumors. Arch Neurol 40:637-642, 1983
9. Lascelles BD, Dernell WS, Correa MT, Lafferty M, Devitt CM, Kuntz CA, et al: Improved survival associated with postoperative wound infection in dogs treated with limb-salvage surgery for osteosarcoma. Ann Surg Oncol 12:10731083,2005

10. Lawthers AG, McCarthy EP, Davis RB, Peterson LE, Palmer RH, Iezzoni LI: Identification of in-hospital complications from claims data. Is it valid? Med Care 38:785-795, 2000

11. Margolis J, West D: Spontaneous regression of malignant disease: report of three cases. J Am Geriatr Soc 15:251253, 1967

12. Naganuma H, Sasaki A, Satoh E, Nagasaka M, Isoe S, Nakano S, et al: Long-term survival in a young patient with anaplastic glioma. Brain Tumor Pathol 14:71-74, 1997

13. Pennica D, Nedwin GE, Hayflick JS, Seeburg PH, Derynck R, Palladino MA, et al: Human tumour necrosis factor: precursor structure, expression and homology to lymphotoxin. Nature 312:724-729, 1984

14. Rahbar A, Stragliotto G, Orrego A, Peredo I, Taher C, Willems J, et al: Low levels of human cytomegalovirus infection in glioblastoma multiforme associates with patient survival; -a case-control study. Herpesviridae 3:3, 2012

15. Ruckdeschel JC, Codish SD, Stranahan A, McKneally MF: Postoperative empyema improves survival in lung cancer. Documentation and analysis of a natural experiment. N Engl J Med 287:1013-1017, 1972

16. Söderberg-Nauclér C, Rahbar A, Stragliotto G: Survival in patients with glioblastoma receiving valganciclovir. N Engl J Med 369:985-986, 2013

17. Stupp R, Mason WP, van den Bent MJ, Weller M, Fisher B, Taphoorn MJ, et al: Radiotherapy plus concomitant and adjuvant temozolomide for glioblastoma. $\mathbf{N}$ Engl J Med 352:987-996, 2005

18. Walid MS: Prognostic factors for long-term survival after glioblastoma. Perm J 12:45-48, 2008

19. Walker DG, Pamphlett R: Prolonged survival and pulmonary metastasis after local cure of glioblastoma multiforme. J Clin Neurosci 6:67-68, 1999

20. Zhang K, Wang XQ, Zhou B, Zhang L: The prognostic value of MGMT promoter methylation in glioblastoma multiforme: a meta-analysis. Fam Cancer 12:449-458, 2013

\section{Disclosures}

The authors report no conflict of interest concerning the materials or methods used in this study or the findings specified in this paper.

\section{Author Contributions}

Conception and design: Skirboll, Chen, Ugiliweneza, Boakye. Acquisition of data: Chen, Ugiliweneza. Analysis and interpretation of data: Chen, Ugiliweneza. Drafting the article: Chen, Ugiliweneza. Critically revising the article: all authors. Reviewed submitted version of manuscript: Skirboll, Chen, Boakye. Statistical analysis: Skirboll, Chen, Boakye. Administrative/technical/ material support: Skirboll, Boakye. Study supervision: Skirboll, Boakye.

\section{Correspondence}

Stephen Skirboll, Department of Neurosurgery, Stanford University Medical Center, 300 Pasteur Dr., R200, Palo Alto, CA 94304. email: skirboll@stanford.edu. 